\title{
New Current-Mode Class 1 Frequency-Agile Filter for Multi Protocol GPS Application
}

\author{
Mesut Atasoyu ${ }^{1}$, Bilgin Metin ${ }^{2}$, Hakan Kuntman ${ }^{1}$, Norbert Herencsar ${ }^{3}$ \\ ${ }^{I}$ Department of Electronics and Communication Engineering, Istanbul Technical University, \\ Maslak, Istanbul, 34469, Turkey \\ ${ }^{2}$ Department of Management Information Systems, Bogazici University, \\ Bebek, Istanbul, 34342, Turkey \\ ${ }^{3}$ Department of Telecommunications, Brno University of Technology, \\ Technicka 3082/12, 61600 Brno, Czech Republic \\ bilgin.metin@boun.edu.tr
}

\begin{abstract}
Recently, due to their cost, accuracy, and integrability of conventional current-mode (CM) on-chip integrated filters working in radio frequency region, frequency-agile filters (FAFs) have started taking great interest in multi-standard transceivers, encrypted communication, cognitive radio, software defined radio structures, and global positioning system applications. By following the most recent trend in the literature, this paper proposes the first class $1 \mathrm{CM}$ FAF using high-performance analog building block so-called positive-type electronically controllable second-generation current conveyor (ECCII+), two resistors, and two grounded capacitors. The theory and the proposed $2^{\text {nd }}$-order CM FAF are supported by both regular and post-layout simulations performed using CADENCE Spectre tool with TSMC $0.18 \mu \mathrm{m}$ level-49 CMOS technology process BSIM3v3 parameters. Furthermore, corner and Monte-Carlo analyses are given to prove the accuracy of centre frequency of the CM FAF.
\end{abstract}

Index Terms-Analog electronics, active filter, current conveyor, ECCII, frequency-agile filter (FAF), tunable circuit, post-layout simulation.

\section{INTRODUCTION}

Electronically tunable filters play important role in several industrial applications. Usually, the centre frequency of tunable filters is changed to compensate the drifts (thermal, technological, etc.) [1]. If the variation of centre frequency is expected to be carried out over a very wide frequency range, reconfigurable filters can be used for compensation. Recently introduced frequency-agile filters (FAFs) by Fabre et al. are special type of reconfigurable filters that have property for agility, i.e. during the transmission of the signal in order not to disturb the signal processing the hop between two consecutive frequencies are able to be carried out very quickly [2] [4]. In general, class $n$ FAFs can be designed by using standard implementation schemes [2]. The main application areas of FAFs are multistandard transceivers (MST), encrypted communication,

Manuscript received January 5, 2015; accepted May 16, 2015.

Research described in this paper was financed by the National Sustainability Program under grant LO1401 and Ing. Norbert Herencsar, Ph.D. was supported by the project CZ.1.07/2.3.00/30.0039 of Brno University of Technology. For the research, infrastructure of the SIX Center was used. This research work is also funded by Bogazici University Research Fund with the project code 08N304. cognitive radio, software defined radio structures, and global positioning systems (GPSs). It is well-known that subblocks of MSTs are low-noise amplifier (LNA), mixer, reconfigurable or adjustable filter, and analog-to-digital converter. Integrated circuit realization of reconfigurable LNAs is not easy to provide even nowadays. Also, RF filters are not easy to reconfigure in integrated form. In transceiver systems, design of reconfigurable structures can be over cost. Similarly, in GPS systems for positioning of different continents, discrete filter structures could be used. Their size, price, complexity and power consumption can be reduced by on-chip integration of FAF. Moreover, architectures' parameters can be still modified in order to be able to adapt to the specifications of different protocols and standards [5]-[7]. Only limited number of FAF topologies using active building blocks (ABBs) working in currentmode (CM) exist in open literature [2], [8]-[10]. It is known that $\mathrm{CM}$ circuits show some advantages against voltagemode circuits such as inherent wider bandwidth, simpler circuitry, lower power consumption, and wider dynamic range [11]. In [2], the intrinsic input resistance $R_{x}$ of currentcontrolled second-generation current conveyor (CCCII) is with advantage used for centre frequency hoping in wide frequency range of class 1 and class 2 band-pass filters. In [8], in order to obtain a CM class $1 \mathrm{FAF}$, the basic class 0 filter employing two current differencing transconductance amplifiers (CDTAs) and two capacitors is extended with electronically controllable second-generation current conveyor (ECCII) functions as amplifier A. In CDTA-based class 1 FAF [9], the $g_{m}$ sub-block of the $2^{\text {nd }}$ CDTA is used for frequency hoping while in [10] additional operational transconductance amplifiers are used as amplifier subcircuits. Moreover, reference [10] also introduces both class 1 and class 2 FAFs employing recently introduced voltage differencing transconductance amplifiers (VDTAs) [12], [13].

By following the most recent trend in the literature, the main aim of this paper is to present a new $\mathrm{CM}$ class $1 \mathrm{FAF}$ using four ECCII+s, two resistors, and two grounded capacitors. The performance of the FAF structure was analyzed using both regular and post-layout simulations in CADENCE Spectre tool. Usually, in integrated filters a 
deviation on the designed filters' centre frequency is significant [14]. In order to prove its accuracy, corner and Monte Carlo (MC) analyses have been also performed.

\section{CIRCUIT DESCRIPTION}

\section{A. Description of FAF and Implementation Schemes}

A FAF is such reconfigurable filter, which has the property of agility, i.e. in order to not disturb the signal processing during the transmission of the signal, the hop between two consecutive frequencies $f_{1}$ and $f_{2}$ must be able to be carried out very quickly [2] [4]. Its implementation is based on such classical $2^{\text {nd }}$-order frequency filter structure, which provides at least band-pass and low-pass responses as it is depicted in Fig. 1. In theory, this $2^{\text {nd }}$-order filter is called as class 0 FAF and the transfer functions $F_{\mathrm{BP} 0}(s)$ and $F_{\mathrm{LP} 0}(s)$ can be expressed as:

$$
\begin{aligned}
& F_{\mathrm{BP} 0}(s)=\frac{I_{\mathrm{BP} 0}}{I_{\mathrm{IN}}}=\frac{a^{\prime} s}{1+a s+b s^{2}}, \\
& F_{\mathrm{LP} 0}(s)=\frac{I_{\mathrm{LP} 0}}{I_{\mathrm{IN}}}=\frac{d^{\prime} s}{1+a s+b s^{2}},
\end{aligned}
$$

where $a$ and $b$ are real positive constants to ensure stability of the filter and $a^{\prime}$ and $d^{\prime}$ are real positive constants that allow to determine the characteristic parameters of the filter.

Class 1 FAF given in Fig. 2 can be obtained from basic $2^{\text {nd }}$-order filter by amplifying the low-pass output current $I_{\mathrm{LP} 0}$ by adjustable gain $A$, which is added to the input current $I_{\mathrm{IN}}$ of previous circuit. Now the new input current of the filter is $I_{\mathrm{E}}$, which is given by the formula $I_{\mathrm{E}}=I_{\mathrm{IN}}-A I_{\mathrm{LP} 0}$. The output $I_{\mathrm{BP} 1}$ remains band-pass response and its corresponding transfer function $F_{\mathrm{BP} 1}(s)$ is

$$
F_{\mathrm{BP} 1}(s)=\frac{I_{\mathrm{BP} 1}}{I_{\mathrm{E}}}=\frac{\frac{a^{\prime} s}{\left(1-A d^{\prime}\right)}}{1+\frac{a s}{\left(1-A d^{\prime}\right)}+\frac{b s^{2}}{\left(1-A d^{\prime}\right)}} .
$$

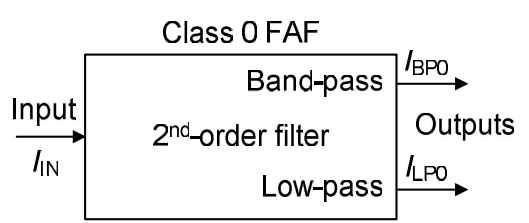

Fig. 1. Basic $2^{\text {nd }}$-order CM filter providing two different outputs.

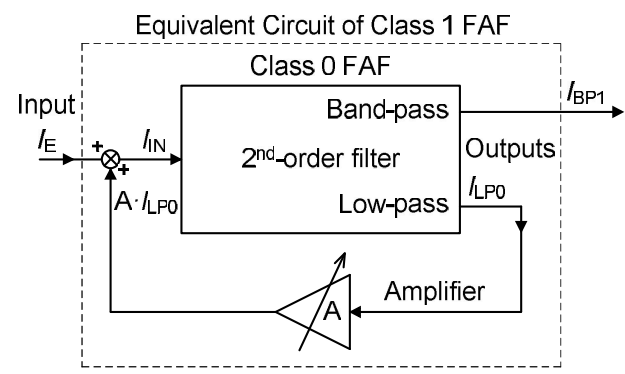

Fig. 2. Class 1 FAF made from the basic $2^{\text {nd }}-$ order filter shown in Fig. 1.

Relationships between the characteristic parameters of both class 0 and class 1 FAFs are listed in Table I. Note that for basic filter (class $0 \mathrm{FAF}$ ), the gains of both outputs will be greater than or equal to unity, i.e. $a^{\prime} \geq a$ and $d^{\prime} \geq 1$. It can be also observed that once the parameters of the starting filters are set, the $f_{0 A}$ of the class 1 FAF can be modified only by the value of gain $A$ of the amplifier. In addition, class 1 FAF will be stable provided that $1 A d^{\prime}$ remains positive.

TABLE I. PARAMETERS OF CLASS 0 AND CLASS 1 FAFS.

\begin{tabular}{|c|c|c|}
\hline & Class 0 FAF & Class 1 FAF \\
\hline Centre frequency & $f_{0}=\frac{1}{2 \pi \sqrt{b}}$ & $f_{0 A}=f_{0} \sqrt{\left(1-A d^{\prime}\right)}$ \\
\hline$Q$-factor & $Q=\frac{\sqrt{b}}{a}$ & $Q_{A}=Q \sqrt{\left(1-A d^{\prime}\right)}$ \\
\hline BP gain & $G_{\mathrm{BP} 0}=\frac{a^{\prime}}{a}$ & $G_{\mathrm{BPA}}=G_{\mathrm{BP} 0}$ \\
\hline BP: $3 \mathrm{~dB}$ bandwidth & $\Delta f=\frac{a}{2 \pi b}$ & $\Delta f_{A}=\Delta f$ \\
\hline LP gain & $G_{\mathrm{LP} 0}=d^{\prime}$ & \\
\hline
\end{tabular}

\section{B. Description of ECCII+}

The ECCII+ is a four-terminal ABB, which circuit symbol and model are shown in Fig. 3 [15], [16]. Using standard notation, the relationships between its port currents and voltages can be described by the following set of equations:

$$
\begin{gathered}
i_{\mathrm{Y}}=0, \\
v_{\mathrm{X}}=v_{\mathrm{Y}}, \\
i_{\mathrm{Z}}=k \times i_{\mathrm{X}} .
\end{gathered}
$$

From (4) (6) and the circuit model in Fig. 3(b) it can be seen that the ECCII+ has a high-impedance (ideally $\infty$ ) voltage input terminal Y, a low-impedance (ideally 0 ) current input terminal $X$, and a high-impedance (ideally $\infty$ ) current output terminal $Z$. Note that the current gain between $\mathrm{X}$ and $\mathrm{Z}$ terminals can be made tunable by the coefficient $k$, which property makes the ECCII+ attractive $\mathrm{ABB}$ for CM FAF design.

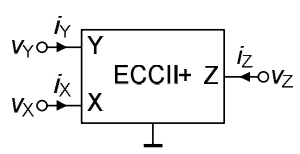

(a)

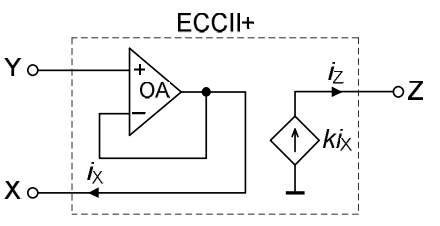

(b)
Fig. 3. Circuit symbol (a), model of ECCII+ (b).

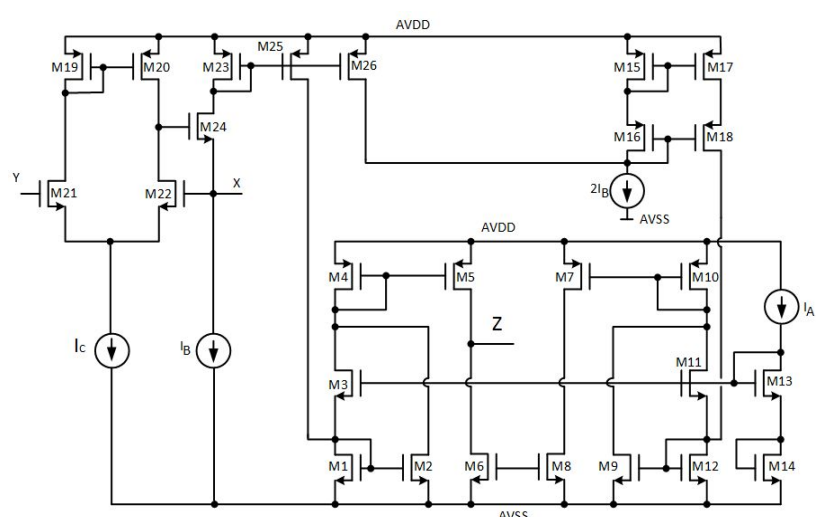

Fig. 4. CMOS ECCII+ internal structure [15]. 
The CMOS implementation of the ECCII+ is shown in Fig. 4 [15], where transistors $\mathrm{M}_{19} \mathrm{M}_{22}$ and current source $I_{\mathrm{C}}$ form a voltage follower stage, which forces the voltage at port $\mathrm{X}$ to follow the voltage at port $\mathrm{Y}$, i.e. $v_{\mathrm{X}} \cong v_{\mathrm{Y}}$. Transistor $\mathrm{M}_{24}$ functions as a current follower stage and also provides a low output resistance at port $\mathrm{X}$. Both stages together can be considered as a grounded voltage-to-current (V/I) converter. For precision $\mathrm{V} / \mathrm{I}$ conversion we have assumed that the pairs of transistors $\mathrm{M}_{21}$ and $\mathrm{M}_{22}, \mathrm{M}_{22}$ and $\mathrm{M}_{24}, \mathrm{M}_{15}$ and $\mathrm{M}_{17}, \mathrm{M}_{16}$ and $\mathrm{M}_{18}$, and $\mathrm{M}_{23}, \mathrm{M}_{25}$, and $\mathrm{M}_{26}$ are well matched, the current mirrors have a unity gain, and all transistors operate in saturation region. The current at the port $\mathrm{Y}$ is zero, i.e. $i_{\mathrm{Y}} \cong 0$, because the input impedance of the MOSFET is very high. Remaining group of transistors $\mathrm{M}_{1}-\mathrm{M}_{14}$ form the output current amplifier stage, which provides current gain tunability between $\mathrm{X}$ and $\mathrm{Z}$ terminals by using current sources $I_{\mathrm{A}}$ and $I_{\mathrm{B}}$ and the output current $i_{\mathrm{Z}}$ can be calculated as

$$
i_{\mathrm{Z}}=n\left(\frac{I_{\mathrm{B}}}{2 I_{\mathrm{A}}}\right) \times i_{\mathrm{X}}=k \times i_{\mathrm{X}},
$$

where $k$ is the small-signal current gain of the amplifier stage and it can be controlled electronically by means of $\mathrm{dc}$ bias currents $I_{\mathrm{A}}$ and $I_{\mathrm{B}}$. Note that the parameter $n$ (current multiplication factor of the current mirrors) can be used to increase the dynamic range of the current gain $k$ to the maximum value $k \leq 2 n$.

The input/output terminal resistances of the CMOS ECCII+ shown in Fig. 4 can be found as:

$$
\begin{gathered}
R_{\mathrm{X}} \simeq \frac{\left(g_{m 21}+g_{m 22}\right)\left(g_{d 20}+g_{d 22}\right)}{g_{m 21} g_{m 22} g_{m 24}}, \\
R_{\mathrm{Y}} \simeq \infty \\
R_{\mathrm{Z}} \simeq \frac{1}{g_{d 5}+g_{d 6}},
\end{gathered}
$$

where $g_{d i}$ and $g_{m i}$ denote the drain transconductance and transconductance of the $i$-th CMOS transistor, respectively.

\section{Proposed ECCII+ Based CM FAF}

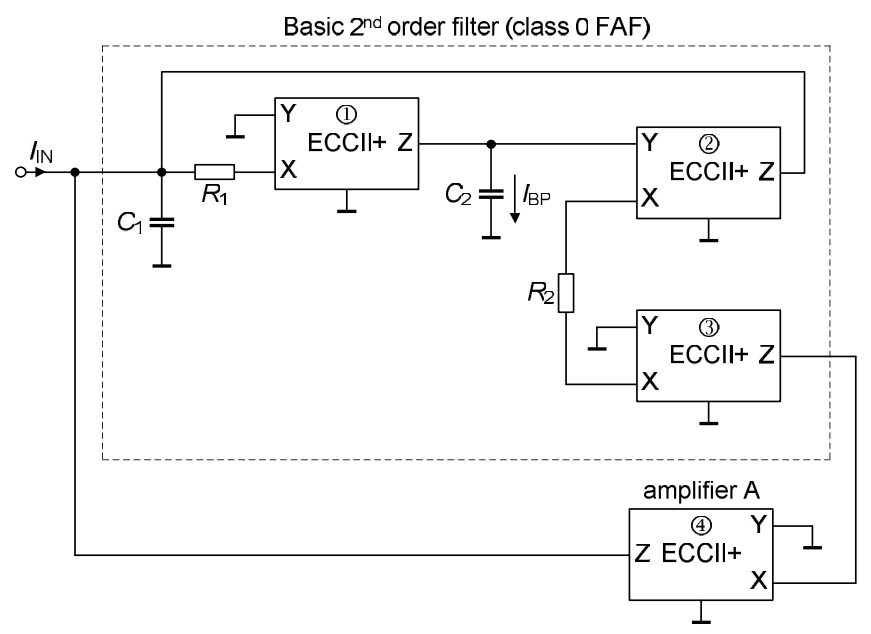

Fig. 5. Proposed frequency-agile filter structure with ECCII+s.

For CM class 1 FAF design the basic $2^{\text {nd }}$-order dual output filter structure in [16] was used. The proposed new filter employing four ECCII+, two resistors, and two capacitors is shown in Fig. 5. In the feedback path the ${ }^{4}$ ECCII+ with tunable coefficient $k_{4}$ functions as an amplifier with tunable gain $A$. Note that this filter corresponds to the case of negative value of $A$. Assuming $k_{1}=k_{2}=k_{3}=1$ and $k_{4}=A$, routine analysis of the circuit gives the following band-pass transfer function $F_{\mathrm{BP} 1}(s)$

$$
F_{\mathrm{BP} 1}(s)=\frac{I_{\mathrm{BP}}}{I_{\mathrm{IN}}}=\frac{\frac{s C_{2} R_{2}}{(1-A)}}{1+\frac{s C_{2} R_{2}}{(1-A)}+\frac{s^{2} C_{1} C_{2} R_{1} R_{2}}{(1-A)}}
$$

The gain $G_{\mathrm{BPA}}$ at $f_{0 A}$ of this band-pass response is unity and the centre frequency $\left(f_{0 A}\right)$ and quality factor $\left(Q_{A}\right)$ of the filter are:

$$
\begin{gathered}
f_{0 A}=\frac{1}{2 \pi} \sqrt{\frac{1}{C_{1} C_{2} R_{1} R_{2}}} \sqrt{(1-A)}, \\
Q_{A}=\sqrt{\frac{C_{1} R_{1}}{C_{2} R_{2}} \sqrt{(1-A)},}
\end{gathered}
$$

which by choosing the capacitors $C_{1}$ and $C_{2}$ and resistors $R_{1}$ and $R_{2}$ identical, i.e. $C_{1}=C_{2}=C, R_{1}=R_{2}=R$, simplify to

$$
f_{0 A}=\frac{1}{2 \pi C R} \sqrt{(1-A)}
$$

and

$$
Q_{A}=\sqrt{(1-A)}
$$

As it can be above seen both the $f_{0 A}$ and $Q_{A}$ change simultaneously by the gain $A$.

\section{Simulation RESUlts}

To verify the theoretical study, the behaviour of the ECCII+ and the new CM $2^{\text {nd }}$-order band-pass class 1 FAF shown in Fig. 4 and Fig. 5 have been verified in CADENCE Spectre design environment with dc power supply voltages equal to $A V_{\mathrm{DD}}=-A V_{\mathrm{SS}}=0.9 \mathrm{~V}$ and bias currents $I_{\mathrm{A}}=37 \mu \mathrm{A}$, $I_{\mathrm{B}}=20 \mu \mathrm{A}$, and $I_{\mathrm{C}}=10 \mu \mathrm{A}$. In the design, transistors were modelled by the TSMC $0.18 \mu \mathrm{m}$ level-49 CMOS technology process BSIM3v3 parameters. In the CMOS ECCII+ structure all transistors" aspect ratio were examined according to dc operating conditions and were selected equally $W / L=24 \mu \mathrm{m} / 0.36 \mu \mathrm{m}$.

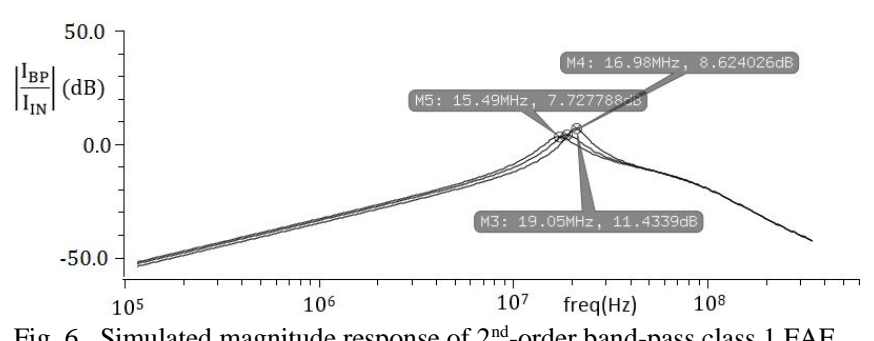

Fig. 6. Simulated magnitude response of $2^{\text {nd }}$-order band-pass class 1 FAF.

First of all, the performance of the ECCII+ was tested by $\mathrm{AC}$ analysis and the maximum operating frequency of the 
ECCII+ was found $f_{\max } \approx 224 \mathrm{MHz}$. Secondly, the performance of the proposed new CM $2^{\text {nd }}$-order band-pass class 1 FAF was verified. In the structure the passive component values were selected as $C_{1}=C_{2}=5 \mathrm{pF}$ and $R_{1}=R_{2}=1 \mathrm{k}$. Figure 6 shows simulated magnitude response of proposed class $1 \mathrm{FAF}$. The centre frequency is varied for $f_{0 A} \cong\{19.05 ; 16.98 ; 15.49\} \mathrm{MHz}$ for bias currents $I_{\mathrm{A}}=\{65 ; 85 ; 95\} \mu \mathrm{A}$, respectively.

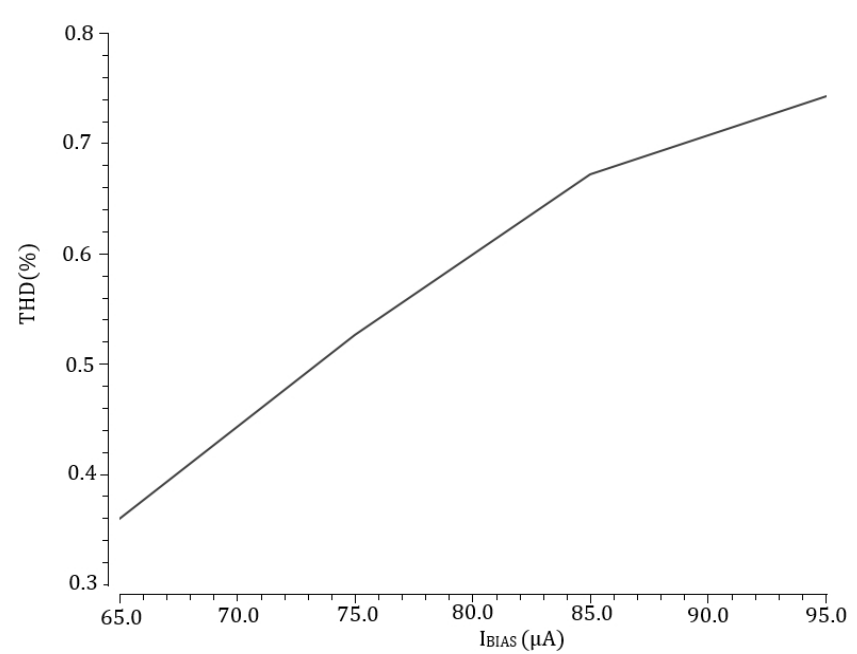

Fig. 7. THD characteristics of band-pass class 1 FAF.

The total harmonic distortion (THD) for the proposed FAF is shown in Fig. 7. During simulations a sinusoidal current input signal with $20 \mathrm{MHz}$ frequency was applied to the FAF while the input current amplitude was changed between $65 \mu \mathrm{A}$ to $95 \mu \mathrm{A}$. The THD values $0.36 \% 0.75 \%$ were obtained with connecting $2 \mathrm{k}$ resistive load to the output.

In order to prove the accuracy of centre frequency $f_{0 A}$, corner and Monte Carlo analyses have been performed. During designing CMOS circuits it is useful to consider conditions of process, voltage, and temperature. In general, process variations can be taken into account since a PMOS is not as fast as a NMOS or vice versa. In corner analysis, this is given as SS, SF, FS, FF (slow-slow, slow-fast, etc.) and/or TT (typical-typical). Also supply voltage variation may occur during operation. Last of all, it is clear that performance can change with temperature. Therefore, the circuits are simulated for performance across the "corner points" of process, voltage, and temperature to validate the circuit operates under all performance conditions. The proposed FAF has also undergone $\mathrm{MC}$ analysis with setting biasing current $I_{\mathrm{A}}=65 \mu \mathrm{A}$, because for this value was achieved the maximum $f_{0 A}$. In corner analysis, the even corners SS, FF, and TT have been considered and positive/negative supply voltage values and the temperature were respectively set $\pm\{0.81$ to 0.99$\} \mathrm{V}$ and $\{50$ to $+120\}^{\circ} \mathrm{C}$. Obtained simulation results are given in Fig. 8. In order to find mean value and standard deviation of centre frequency, the MC analysis histogram is given in Fig. 9. The mean value of $f_{0 A}$ is found as $17.4635 \mathrm{MHz}$ and standard deviation $3.89745 \mathrm{MHz}$, which is close to the $f_{0 A}$ of the designed FAF.

In addition, the performance of the proposed $\mathrm{CM} 2^{\text {nd }}$ order class 1 FAF was verified by post-layout simulations in CADENCE Spectre tool. The layout of the ECCII+ from
Fig. 4 is shown in Fig. 10. Its total area is $1582.22 \mu \mathrm{m}^{2}$.

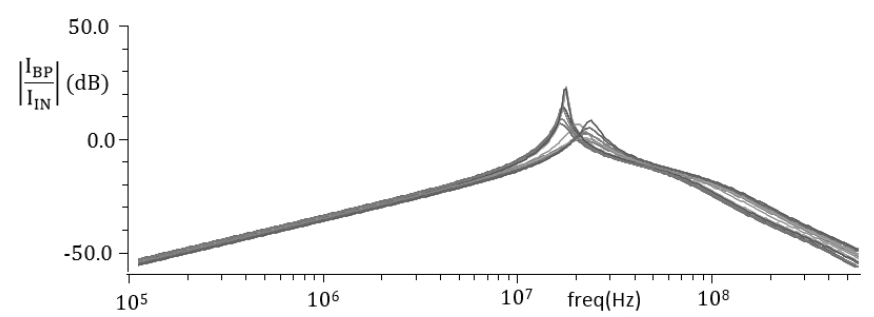

Fig. 8. Corner analysis results of band-pass class 1 FAF.

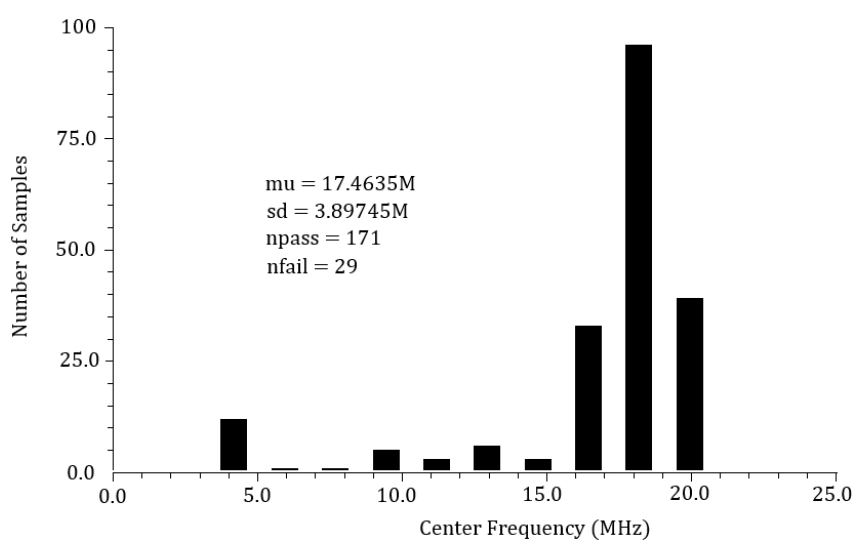

Fig. 9. Monte Carlo analysis histogram of designed band-pass class 1 FAF.

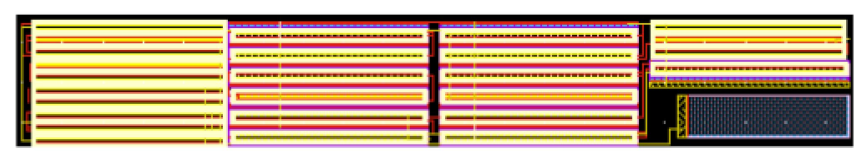

Fig. 10. The layout of ECCII+ circuit structure.

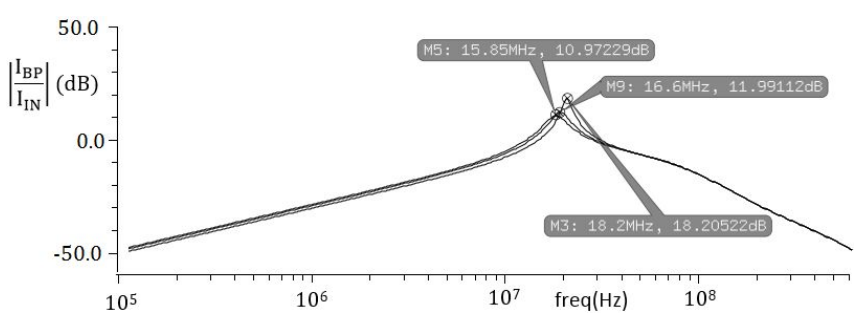

Fig. 11. Post-layout magnitude simulations of band-pass class 1 FAF.

In post-layout simulations of the $2^{\text {nd }}$-order band-pass class 1 FAF the centre frequency is varied for $f_{0 A} \cong\{18.2 ; 16.6$; $15.85\} \mathrm{MHz}$ for bias current $I_{\mathrm{A}}=\{65 ; 85 ; 95\} \mu \mathrm{A}$, respectively, and simulated magnitude responses are shown Fig. 11. It can be pointed out that due to layout parasitics there is no significant difference between regular and postlayout simulation responses.

\section{CONCLUSIONS}

In order to increase the application possibilities of ECCII+, this paper presents a new CM $2^{\text {nd }}$-order band-pass class 1 FAF. Compared to basic $2^{\text {nd }}$-order band-pass filter initially designed for $f_{0}=450 \mathrm{kHz}$ [16], the $f_{0 A}$ of the new FAF is hoping over more than one decade higher - up to $19.05 \mathrm{MHz}$ or $18.2 \mathrm{MHz}$ in regular or post-layout simulations, respectively. In post-layout simulations with setting maximum biasing current $I_{\mathrm{A}}$ the total power dissipation of FAF was determined as $8.17 \mathrm{~mW}$.

\section{REFERENCES}

[1] L. T. Bruton, RC-Active Circuits Theory and Design. Prentice-Hall: Englewood Cliffs, NJ, 1980. 
[2] Y. Lakys, B. Godara, A. Fabre, "Cognitive and encrypted communications: state of the art and a new approach for frequencyagile filters", Turkish Journal of Electrical Engineering \& Computer Sciences, vol. 19, no. 2, pp. 251-273, 2011. [Online] Available: http://dx.doi.org/10.3906/elk-1001-374

[3] Y. Lakys, A. Fabre, "Multistandard transceivers: state of the art and a new versatile implementation for fully active frequency agile filters", Analog Integrated Circuits and Signal Processing, vol. 74, no. 1, pp. 63-78, 2013. [Online] Available: http://dx.doi.org/10.1007/s10470-012-9861-4

[4] Y. Lakys, A. Fabre, "Encrypted communications: towards very low consumption frequency-hopping active filters", Analog Integrated Circuits and Signal Processing, vol. 81, no. 1, pp. 5-16, 2014. [Online] Available: http://dx.doi.org/10.1007/s10470-014-0344-7

[5] P.-I. Mak, S.-P. U, R. P. Martins, "Transceiver architecture selection: Review, state-of-the-art survey and case study", IEEE Circuits and Systems Magazine, vol. 7, no. 2, pp. 6-25, 2007. [Online] Available: http://dx.doi.org/10.1109/MCAS.2007.4299439

[6] J. Mitola, "The software radio architecture", IEEE Communications Magazine, vol. 33, no. 5, pp. 26-38, 1995. [Online] Available: http://dx.doi.org/10.1109/35.393001

[7] N. Qi, Y. Xu, B. Chi, X. Yu, X. Zhang, N. Xu, P. Chiang, W. Rhee, Z. Wang, "A dual-channel compass/GPS/GLONASS/Galileo reconfigurable GNSS receiver in $65 \mathrm{~nm}$ CMOS with on-chip I/Q calibration", IEEE Trans. Circuits and Systems I, vol. 59, no. 8, pp. 1720-1732, 2012. [Online] Available: http://dx.doi.org/10.1109/ TCSI.2012.2206502

[8] E. Alaybeyoglu, A. Guney, M. Altun, H. Kuntman, "Design of positive feedback driven current-mode amplifiers Z-Copy CDBA and CDTA, and filter applications", Analog Integrated Circuits and Signal
Processing, vol. 81, no. 1, pp. 109-120, 2014. [Online] Available: http://dx.doi.org/10.1007/s10470-014-0345-6

[9] N. Pandey, R. Pandey, R. Choudhary, A. Sayal, M. Tripathi, "Realization of CDTA based frequency Agile filter", in Proc. (ISPCC 2013), Solan, India, 2013, pp. 1-6. [Online] Available: http://dx.doi.org/10.1109/ISPCC.2013.6663403

[10] N. Pandey, A. Sayal, R. Choudhary, R. Pandey, "Design of CDTA and VDTA based frequency Agile filters", Advances in Electronics, vol. 2014, p. 15, 2014. [Online] Available: http://dx.doi.org/10.1155/ $2014 / 176243$

[11] C. Toumazau, F. J. Lidgey, D. G. Haig, Analogue IC Design: The Current-Mode Approach. London: Peter Peregrinus Ltd., 1990.

[12] D. Biolek, R. Senani, V. Biolkova, Z. Kolka, "Active elements for analog signal processing: classification, review, and new proposals", Radioengineering, vol. 17, no. 4, pp. 15-32, 2008.

[13] N. Herencsar, R. Sotner, J. Koton, J. Misurec, K. Vrba, "New compact VM four-phase oscillator employing only single z-copy VDTA and all grounded passive elements", Elektronika ir Elektrotechnika, vol. 19, no. 10, pp. 87-90, 2013. [Online] Available: http://dx.doi.org/ 10.575/j01.ee.19.10.5900

[14] Y. Tsividis, "Continuous-time filters in telecommunications chips", IEEE Communications Magazine, vol. 39, no. 4, pp. 132-137, 2001 [Online] Available: http://dx.doi.org/10.1109/35.917515

[15] W. Surakampontorn, K. Kumwachara, "CMOS-based electronically tunable current conveyor", Electron. Lett., vol. 28, pp. 1316-1317, 1992. [Online] Available: http://dx.doi.org/10.1049/el: 19920836

[16] S. Minaei, O. K. Sayin, H. Kuntman, "A new CMOS electronically tunable current conveyor and its application to current-mode filters", IEEE Trans. Circuits and Systems I, vol. 53, pp. 1448-1457, 2006. [Online] Available: http://dx.doi.org/10.1109/TCSI.2006.875184 